\title{
RAPID DETECTION OF MULTI DRUG RESISTANCE AMONG MULTI DRUG RESISTANT TUBERCULOSIS SUSPECTS USING LINE PROBE ASSAY
}

\author{
MANOJ KUMAR DUBEY ${ }^{1}$, UMA BHARDWAJ ${ }^{1}$, M HANIF ${ }^{2 *}$, CHOPRA KK ${ }^{2}$, ASHWANI KHANNA ${ }^{3}$, SANJEEV SAINI ${ }^{2}$, \\ KAUSHAL KUMAR DWIVEDI ${ }^{2}$, HIMANSHU VASHISTHA ${ }^{2}$, ZEESHAN SIDIQ ${ }^{2}$, VASIM AHMED ${ }^{2}$, SRASHTY SHARMA ${ }^{2}$
}

${ }^{1}$ Department of Microbiology, Maharaja Vinayak Global University, Jaipur, Rajasthan, India. ${ }^{2}$ Laboratory section, New Delhi Tuberculosis Centre, New Delhi, India. ${ }^{3}$ State TB Program Officer, New Delhi, India. Email: irldlndc@rntcp.org

Received: 27 July 2016, Revised and Accepted: 27 September 2016

\section{ABSTRACT}

Objective: GenoType MTBDRplus line probe assay (LPA) is developed for performing drug susceptibility testing (DST) for Rifampicin (RIF) and isoniazid in sputum specimens from smear-positive pulmonary tuberculosis (TB) patients and revised national TB control Programme (RNTCP) has endorsed LPA for the diagnosis of multi drug resistant TB (MDR-TB). This study was conducted to assess the potential utility of LPA for MDR-TB patient management.

Methods: MDR-TB suspects under RNTCP PMDT criteria C referred from different districts in Delhi state were included in the study January 2013 to December 2014. Sputum specimens found acid-fast bacilli positive by fluorescent microscopy were processed for LPA.

Results: Out of 3062 specimens, 2055 (67.1\%) MDR-TB suspects were read as positive and specimens from 1007 (32.9\%) suspects were read as negative in sputum smear microscopy. Out of 2019 specimens valid LPA results, 1427 were found to be pan-sensitive, 280 were MDR-TB, 40 were RIF monoresistant, 183 were Isoniazid (INH) monoresistant, and 89 specimens were found negative for Mycobacterium tuberculosis.

Conclusion: Routine use of LPA can substantially reduce the time to diagnosis of RIF and/or INH-resistant TB and can hence potentially enable earlier commencement of appropriate drug therapy and thereby facilitate prevention of further transmission of drug resistant strains.

Keywords: Multi drug resistant tuberculosis, Line probe assay, Rifampicin, Isoniazid.

(C) 2017 The Authors. Published by Innovare Academic Sciences Pvt Ltd. This is an open access article under the CC BY license (http://creativecommons. org/licenses/by/4. 0/) DOI: http://dx.doi.org/10.22159/ajpcr.2017.v10i1.14341

\section{INTRODUCTION}

Rapid determination of drug resistance can allow a customized approach to treatment early in the course of the disease and can potentially reduce morbidity, mortality, and infectiousness [1]. The World Health Organization and partners have proposed a Global XDR-TB response plan [2], which calls for wide-scale implementation of rapid methods to screen patients at risk of MDR-TB. Rapid tests can provide results within days and thus enable prompt and appropriate treatment, decrease morbidity and mortality and interrupt transmission.

The identification of specific mutations responsible for drug resistance has facilitated the development of novel, rapid molecular tools for drug susceptibility testing (DST). The detection of RIF resistance is traditionally used as a predictor of MDR-TB - its positive predictive value is a function of the sensitivity and specificity of RIF resistance testing and the prevalence of MDR and non-MDR RIF resistance, which is highest among previously treated cases in settings with high MDR prevalence and low non-MDR RIF resistance. Molecular tools are based on nucleic acid amplification in conjunction with electrophoresis, sequencing, or hybridization.

Reverse hybridization-based assays, referred to as line probe assays, represent a useful tool for their superior cost-effectiveness. These tests are based on the hybridization of specific probes for wild-type and mutated sequences of genes involved in drug resistance, and they show high specificity and medium/high sensitivity.

GenoType MTBDRplus (Hain Lifescience, Germany) enables the simultaneous molecular genetic identification of the M. tuberculosis complex and its resistance to Rifampicin (RIF) and isoniazid from clinical specimens or cultivated samples. The benefits of using GenoType MTBDRplus can detect M. tuberculosis-specific DNA and genetic mutations associated with drug resistance from smear-positive sputum specimens or culture isolates, after DNA extraction and PCR amplification [3]. The use of LPAs has been recommended by the WHO [4].

Revised national TB control Programme has also endorsed the LPA MTBDRplus, Hain Lifesciences, Germany for the diagnosis of MDR-TB. This assay is being used now as screening test across the country for all suspects of MDR-TB.

\section{METHODS}

This study was conducted at the Laboratory of New Delhi TB Centre (NDTB), New Delhi from February 2014 to December 2015.

The laboratory is certified by Central TB division, Ministry of Health and Family Welfare, Government of India for conducting liquid, solid culture and DST and LPA.

\section{Study population}

All MDR-TB suspects referred to New Delhi TB center, New Delhi during the period from January 2013 to December 2014 from 17 Chest Clinics of Delhi were included in the study.

\section{Specimen collection}

Samples from a total of three thousand and sixty two $(n=3062)$ MDR-TB suspects were included in the study. All these cases were enrolled at different chest clinics of Delhi. Two sputum samples (spot and morning) were collected per patient in $50 \mathrm{ml}$ sterile conical centrifuge tubes and were transported on same day along with duly filled request form for Culture and DST to New Delhi TB centre. On receipt, these specimens were checked for any spill or leakage. Spilled or leaked specimens, as well as inadequate quantity specimens, were rejected, and all others 
were accepted for processing. In all these specimens, quality was examined by visual appearance. Sputum samples from 2431 (79.4\%) suspects were found Mucoid/Mucopurulent, 227 (7.42\%) were blood stained, and $404(13.18 \%)$ were saliva.

\section{Sputum smear microscopy}

All manipulations with potentially infectious clinical specimens were performed in a Class II safety cabinet in a BSL-3 Laboratory. Smears were prepared from all these specimens and were subjected to Auramine staining. They were examined under $\times 400$ magnification using a LED fluorescence microscope and graded according to WHO/ IUATLD guidelines [5].

Sputum specimens were processed using N-acetyl-cysteine-sodium hydroxide- $\mathrm{NaOH}$ decontamination $(\mathrm{NaOH}$ final concentration, $1.5 \%$ ) [6]. Following centrifugation, the pellet in each tube was suspended in $2.5 \mathrm{ml}$ of phosphate buffer $\mathrm{pH}$ 6.8. Processed sediments from the same patient were pooled and mixed thoroughly. One portion of the sediment was used for LPA and another portion was used for culture in LJ media.

\section{LPA}

LPA testing was performed in three separate rooms, according to WHO recommendations. DNA extraction was performed in the BSL3 laboratory. Master mix preparation, PCR, and hybridization were performed in separate rooms.

About $500 \mu \mathrm{l}$ of processed sediment was used to perform the Genotype MTBDRplus (Hain Lifescience $\mathrm{GmbH}$ ) assay, according to the manufacturer's instructions [7]. After observation of final band pattern depending on the presence or absence of bands, the results were interpreted as sensitive or resistant.

LPA testing on samples with invalid results was repeated using the stored residual extracted DNA. To consider a band valid for study purposes, the band intensity had to be equal or greater than the AC band (according to the product insert).

\section{RESULTS}

Specimens from 2055 (67.1\%) MDR-TB suspects were read as positive and specimens from 1007 (32.9\%) suspects were read as negative. Statistically significant difference was observed between negative and positive specimens among different types of sputum specimens as depicted by $\mathrm{p}$ value (Table 1 ).

During observation under microscope, grading was done in addition to identification of positive results grading of smear-positive sputum specimens is detailed in Table 2 . Although majority of the specimens were graded as $1+$ or $2+$ among different types of specimens, no significant difference in $\mathrm{p}$ value was observed while comparing grading of smears.

Among the positive smears $(\mathrm{n}=2055), 304$ (14.8\%) were read as scanty, $685(33.3 \%)$ were read as $1+, 824(40.1 \%)$ were $2+$ and $242(11.8 \%)$ were $3+$.

Out of 2055 smear positive specimens processed through LPA, valid results were found in 2019, remaining were indeterminate/invalid.
Of $2019,69.4 \%$ were found to be pan-sensitive, $11.7 \%$ were MDR-TB, $3.9 \%$ were RIF monoresistant, $8.9 \%$ were INH monoresistant, and $4.3 \%$ specimens were found negative for M. tuberculosis (Table 3).

\section{DISCUSSION}

The routine use of LPA can substantially reduce the time to diagnosis of RIF and/or INH-resistant TB and can hence potentially enable earlier commencement of appropriate drug therapy and thereby facilitate prevention of further transmission of drug-resistant strains. This confers a major advantage to this test. Therefore, this study was conducted using the LPA for the diagnosis of MDR-TB among the MDRTB suspects.

The overall proportion of interpretable results with the Genotype MTBDRplus assay (92\%) was significantly higher when compared to conventional methods (78\%). The assay gave interpretable results in a considerable number of specimens (99) in which no conventional DST result was available (mostly due to contamination of the primary LJ culture), and detected an additional 09 MDR-TB cases.

Of the total valid LPA tests, MDR pattern was observed among $280(11.7 \%)$ specimens, Mono RIF resistance among $40(3.9 \%)$ and mono isoniazid resistance in 183 (8.9\%) specimens. These observations are in alliance with the national level data obtained from multiple laboratories. The performance of the LPA in this setting was similar to that reported previously, with high specificity for detection of RIF and isoniazid, high sensitivity for detection of RIF resistance, and isoniazid resistance [8].

It was observed in this study that RIF resistance was highly associated with mutation in the 81 base pair region of the $r p o B$ gene. This is in agreement with the results published earlier $[9,10]$. In addition, our observation that Isoniazid resistance was most commonly associated with katG mutation was similar to the one reported earlier [11].

LPA invalid results were obtained in 7 samples and indeterminate results were obtained in 29 samples. Of these 36 samples, 28 were scanty positive and 20 were culture negative. This proves that this test is not suitable for smear-negative clinical specimens [12,4]. It was previously reported that low-grade smear positivity correlated to invalid results in LPA [13].

In our laboratory, around 700 specimens are processed monthly and the workflow is adjusted in a way that the final results of the test should be available within the turn-around-time (TAT). Early detection of MDR or RIF resistant by LPA led to the early initiation of MDR treatment. Before the introduction of LPA in Delhi state, only $70 \%$ of diagnosed MDRTB cases by conventional LJ culture and DST were put on treatment, and the remaining cases were either died or defaulted. But after the introduction of LPA, more than $90 \%$ of diagnosed cases were started on treatment (unpublished data). However, the impact of reduced TAT on patient outcomes such as sputum culture conversion, cure, mortality, and treatment failure also remains to be assessed but it is conceivable that early diagnosis of MDR-TB together with early treatment initiation will have a significant impact at the patient in public health level.

It was reported earlier that overall the cost of performing the MTDBDRplus test was lower when the assay is performed directly on

Table 1: Direct AFB fluorescent microscopy results among different types of sputum specimens

\begin{tabular}{llll}
\hline Quality of sputum & $\begin{array}{l}\text { Number of MDR-TB } \\
\text { suspects (n=3062) }\end{array}$ & Smears microscopy result & p (Chi-square test) \\
\cline { 3 - 4 } & & Negative (n=1007) n (\%) & Positive (n=2055) n (\%) \\
\hline Mucoid & 2431 & $678(67.3)$ & $1753(85.3)$ \\
Bloody & 227 & $80(7.9)$ & $147(7.2)$ \\
Saliva & 404 & $249(24.7)$ & $155(7.5)$ \\
\hline
\end{tabular}

MDR-TB: Multi drug resistant tuberculosis

Table 2: Grading of smear positive sputum specimens

\begin{tabular}{|c|c|c|c|c|c|c|}
\hline \multirow[t]{2}{*}{ Quality of sputum } & \multicolumn{4}{|c|}{ Grading of positive smear n (\%) } & \multirow[t]{2}{*}{ Total positive $(n=2055)$} & \multirow[t]{2}{*}{ p (Chi-square test) } \\
\hline & Scanty $(n=304)$ & $1+(n=685)$ & $2+(n=824)$ & $3+(n=242)$ & & \\
\hline Mucoid & $242(79.55)$ & $590(86.49)$ & $725(88.02)$ & $196(80.95)$ & 1753 & 0.837 \\
\hline Saliva & $34(11.36)$ & $43(6.49)$ & $55(6.59)$ & $23(9.52)$ & 155 & 0.856 \\
\hline
\end{tabular}


Table 3: LPA results among smear positive specimens

\begin{tabular}{ll}
\hline LPA results & Number (\%) $\mathbf{n = 2 0 5 5}$ \\
\hline Sensitive & $1427(69.4)$ \\
MDR-TB & $280(11.7)$ \\
RIF monoresistant & $40(3.9)$ \\
INH monoresistant & $183(8.9)$ \\
MTBC negative & $89(4.3)$ \\
Invalid & $7(0.3)$ \\
Indeterminate & $29(1.4)$ \\
\hline
\end{tabular}

LPA: Line probe assay, MDR-TB: Multi drug resistant tuberculosis, RIF: Rifampicin, INH: Isoniazid

smear-positive sputum specimens $[14,15]$. In our study, it was not possible to do costing analysis due to various factors like support by the government in the form of new equipment and supplies in addition to fulfillment of manpower.

The availability of valid LPA results proved the advantages of molecular test over the conventional method.

\section{REFERENCES}

1. Hopewell PC, Pai M, Maher D, Uplekar M, Raviglione MC. International standards for tuberculosis care. Lancet Infect Dis 2006;6(11):710-25.

2. World Health Organization. The Global MDR TB and XDR TB Response Plan, 2007-2008. WHO/HTM/TB/2007, 387. Geneva: World Health Organization; 2007.

3. Abebe G, Paasch F, Apers L, Rigouts L, Colebunders R. Tuberculosis drug resistance testing by molecular methods: Opportunities and challenges in resource limited settings. J Microbiol Methods 2011;84(2):155-60.

4. World Health Organization. Policy Statement. Molecular Line Probe Assays for Rapid Screening of Patients at Risk of Multidrug Resistant Tuberculosis (MDR-TB), 2008. Available from: http://www.who.int/tb/ dots/laboratory/lpa policy.pdf. [Last accessed on 2009 Nov 18].

5. World Health Organization. WHO Laboratory Services in Tuberculosis
Control. Part II: Microscopy. WHO/TB/98, 258. Geneva, Switzerland: WHO; 1998.

6. Master RN. Mycobacteriology. In: Isenburg HD, editor. Clinical Microbiology Procedures Handbook. Section 3. Washington, DC: American Society for Microbiology; 1992. p. 1.

7. HainLifescience. Genotype ${ }^{\circledR}$ MTBDRplus product insert. Version 1. Available from: http:/www.hainlifescience.de/en/products/ microbiology/mycobacteria/genotypemtbdrplus.

8. Ling DI, Zwerling AA, Pai M. GenoType MTBDR assays for the diagnosis of multidrug-resistant tuberculosis: A meta-analysis. Eur Respir J 2008;32(5):1165-74.

9. Telenti A, Imboden P, Marchesi F, Lowrie D, Cole S, Colston MJ, et al. Detection of rifampicin-resistance mutations in Mycobacterium tuberculosis. Lancet 1993;341(8846):647-50.

10. Van Rie A, Warren R, Mshanga I, Jordaan AM, van der Spuy GD, Richardson $\mathrm{M}$, et al. Analysis for a limited number of gene codons can predict drug resistance of Mycobacterium tuberculosis in a highincidence community. J Clin Microbiol 2001;39(5):636-41.

11. Mokrousov I, Narvskaya O, Otten T, Limeschenko E, Steklova L, Vyshnevskiy B. high prevalence of KatG ser315Thr substitution among isoniazid-resistant Mycobacterium tuberculosis clinical isolates from northwestern Russia, 1996 to 2001. Antimicrob Agents Chemother 2002;46(5):1417-24.

12. Dorman SE, Chihota VN, Lewis JJ, van der Meulen M, Mathema B, Beylis $\mathrm{N}$, et al. Genotype MTBDRplus for direct detection of Mycobacterium tuberculosis and drug resistance in strains from gold miners in South Africa. J Clin Microbiol 2012;50(4):1189-94.

13. Barnard M, Albert H, Coetzee G, O’Brien R, Bosman ME. Rapid molecular screening for multidrug-resistant tuberculosis in a highvolume public health laboratory in South Africa. Am J Respir Crit Care Med 2008;177(7):787-92.

14. Morgan M, Kalantri S, Flores L, Pai M. A commercial line probe assay for the rapid detection of rifampicin resistance in Mycobacterium tuberculosis: A systematic review and meta-analysis. BMC Infect Dis 2005;5:62.

15. World Health Organization. New Laboratory Diagnostic Tools for Tuberculosis Control. Stop TB Partnership: Retooling Task Force and the New Diagnostics Working Group. Geneva, Switzerland: World Health Organization; 2008. 